\title{
Epidemiological Impact of Tenofovir Gel on the HIV Epidemic in South Africa
}

\author{
Brian G. Williams, PhD, * Salim S. Abdool Karim, MBChB, PhD, † \\ Quarraisha Abdool Karim, PhD, $\uparrow$ and Eleanor Gouws, PhD $\neq$
}

\begin{abstract}
Background: Tenofovir gel, an antiretroviral-based vaginal microbicide, reduced HIV acquisition by $39 \%$ in women in a recent randomized controlled clinical trial in South Africa.
\end{abstract}

\begin{abstract}
Methods: To inform policy, we used a dynamical model of HIV transmission, calibrated to the epidemic in South Africa, to determine the population-level impact of this microbicide on HIV incidence, prevalence, and deaths and to evaluate its cost-effectiveness.
\end{abstract}

Results: If women use tenofovir gel in $80 \%$ or more of sexual encounters (high coverage), it could avert 2.33 ( 0.12 to 4.63 ) million new infections and save 1.30 ( 0.07 to 2.42) million lives and if used in $25 \%$ of sexual encounters (low coverage), it could avert 0.50 (0.04 to 0.77 ) million new infections and save 0.29 ( 0.02 to 0.44 ) million deaths, over the next 20 years. At US $\$ 0.50$ per application, the cost per infection averted at low coverage is US \$2392 (US \$562 to US \$4222) and the cost per disability-adjusted life year saved is US \$104 (US \$27 to US \$181); at high coverage the costs are about $30 \%$ less.

Conclusions: Over 20 years, the use of tenofovir gel in South Africa could avert up to 2 million new infections and 1 million AIDS deaths. Even with low rates of gel use, it is highly cost-effective and compares favorably with other control methods. This female-controlled prevention method could have a significant impact on the epidemic of HIV in South Africa. Programs should aim to achieve gel use in more than $25 \%$ of sexual encounters to significantly alter the course of the epidemic.

Key Words: HIV prevention, microbicide gel, tenofovir, South Africa, cost effectiveness

(J Acquir Immune Defic Syndr 2011;58:207-210)

Received for publication March 4, 2011; accepted May 4, 2011.

From the *South African Centre for Epidemiological Modelling and Analysis (SACEMA), Stellenbosch, South Africa; †Center for the AIDS Program of Research in South Aftrica (CAPRISA), University of KwaZulu-Natal, South Africa and Department of Epidemiology, Columbia University, New York; and $\ddagger$ Strategic Intelligence and Analysis Division, UNAIDSJoint United Nations Program on HIV/AIDS, Geneva, Switzerland.

The authors have no funding or conflicts of interest to disclose.

All authors contributed to the design of the study, the interpretation of the data, and writing of the article. B.G.W. and E.G. carried out the modelling.

Correspondence to: Brian Gerard Williams, PhD, South African Centre for Epidemiological Modelling and Analysis (SACEMA), 19 Jonkershoek Road, Stellenbosch, South Africa (e-mail: briangerardwilliams@gmail. com).

Copyright (C) 2011 by Lippincott Williams \& Wilkins

\section{INTRODUCTION}

The global HIV epidemic continues unabated, and despite recently observed declines in HIV incidence among young women in some countries in Africa, incidence rates remain unacceptably high. ${ }^{1}$ Efforts to reduce HIV transmission have yet to show a significant population level effect in many countries in Africa. ${ }^{2}$ The evidence that treating sexually transmitted infections reduces HIV incidence remains equivocal; ${ }^{3}$ behavior change interventions have not shown a demonstrable impact on HIV incidence ${ }^{4}$ male circumcision reduces the risk of infection by about $60 \%$ in men and is now being made available in much of Africa. ${ }^{5}$ A recent study reports that male circumcision might reduce HIV infection directly in women, but the reduction was not statistically significant in the trials quoted. ${ }^{6}$ Condoms are effective, if used consistently and correctly, and promoting condoms among sex workers has yielded positive results, but condom use remains limited. ${ }^{7}$ Where it is available, antiretroviral treatment has reduced mortality rates, but there is little evidence that it has significantly reduced community-level transmission because it is currently provided mainly in the late stages of infection. ${ }^{8}$ There is therefore an urgent need for new prevention technologies that women can use to reduce their risk of infection.

In the Centre for the AIDS Program of Research in South Africa (CAPRISA) 004 randomized controlled trial, a vaginal microbicide containing $1 \%$ tenofovir significantly reduced the transmission of HIV- $1^{9}$ from men to women. The availability of this female-controlled method of prevention could have a significant impact on the future epidemic of HIV. In countries like South Africa, where the HIV epidemic is generalized and incidence rates among young women are high, ${ }^{10,11}$ policies on tenofovir gel are being developed for post-licensure implementation. To help inform policy development and resource allocation, we estimate the potential long-term impact of tenofovir gel on HIV prevalence, HIV incidence, and AIDS mortality in South Africa and its cost-effectiveness.

\section{METHODS}

A mathematical model, ${ }^{12}$ previously developed to explore the impact of male circumcision on the HIV epidemic, has been adapted for this study. Briefly, we compartmentalize the population into 5 groups: people who are not infected with HIV and people in 4 stages of HIV infection. We assume a mean survival time from HIV infection to death, without antiretroviral treatment, of 11 years and let transmission rates fall as prevalence rises to allow for heterogeneity in sexual risk behavior as 
discussed fully in our previous study. ${ }^{12}$ We fitted the model to trends in the prevalence of HIV among adults as estimated by the Joint United Nations Program on HIV/AIDS. ${ }^{2}$ The model is not structured by age or gender. Assuming that the microbicide reduces male-to-female transmission with a relative risk of infection $r$, say, but does not reduce female-to-male transmission, then this is equivalent to a reduction in overall transmission with a relative risk of $r *=\sqrt{r}{ }^{12}$ We have previously shown that the long-term population level impact using this model, without stratifying by gender, is not significantly different from the impact calculated using a model that is stratified by gender. ${ }^{12}$

We consider 3 coverage scenarios, where coverage is defined as the proportion of all sexual encounters that are protected by the use of the gel. "High" is for women who used the gel in $80 \%$ or more of sexual contacts in the CAPRISA 004 trial $^{9}$ for which the relative risk of infection was $r_{h}=0.46(0.20$ to 0.96 ; limits are $95 \%$ confidence intervals here and elsewhere). We assume that the average coverage in the "high" scenario was $90 \%$. If the relative risk of infection per sexual encounter protected by the gel is $r$ and the coverage is $c$ then the average risk, at coverage $c$, is:

$$
r_{c}=r c+(1-c)
$$

Setting $c=0.90$ and $r_{c}=r_{h}$ gives $r=0.40$ ( 0.11 to 0.96$)$. For the "medium" scenario, we assume that coverage is $50 \%$ so that $r_{m}=0.70(0.56$ to 0.98$)$ and for the low scenario that coverage is $25 \%$ so that $r_{l}=0.85$ ( 0.78 to 0.99 ) (equation 1$)$. In short, based on the CAPRISA 004 trial results, we assume $90 \%$ (high) coverage achieves $60 \%$ protection from HIV, $50 \%$ (medium) coverage achieves $30 \%$ protection, and $25 \%$ (low) coverage achieves $15 \%$ protection.

We let the cost-effectiveness, $C$, be the cost of using the microbicide divided by the reduction in the incidence of infection. If the cost per dose is $\gamma$, the coverage is $c$, and susceptible women, who use the microbicide, have $\eta$ sexual contacts per year, then the annual cost is $\gamma \eta c$. We let the incidence of HIV infection among susceptible women be $I$, and the reduction in the risk of infection resulting from the use of the microbicide be $\rho_{c}=1-\sqrt{r_{c}}$ In nonlinear epidemiological models, when the epidemic is far from elimination, the proportional reduction in incidence is generally less than the proportional reduction in transmission by an amount $\phi$, say, which will depend on the natural history of the epidemic and the structure of the model that is used to fit the data. ${ }^{13}$ The annual incidence of HIV, per susceptible woman, is then $\phi \rho I$, so that the cost per infection averted is:

$$
c=\frac{\gamma \eta c}{\phi \rho I}
$$

If averting one infection saves $\delta$ years of disabilityadjusted life, the cost per disability adjusted life-year (DALY) saved is:

$$
D=\frac{c}{\delta}
$$

Verguet et $\mathrm{al}^{14}$ suggested that the cost of the microbicide in a developing country is US \$0.50. In the CAPRISA 004 Trial, ${ }^{9}$ the cost of the gel was US $\$ 0.01$, the tenofovir was donated free for the trial but is likely to cost about US $\$ 0.02$, whereas the cost of the applicator was US \$ 0.11 and of the wrapping US \$ 0.16 , giving a total cost for 2 applications ( 1 before and 1 after the sexual encounter) of $\gamma=$ US $\$ 0.60$. Large-scale production of the gel and cheaper applicators could reduce this cost further. For the purposes of this analysis, the full trial production costs of the product are used. Verguet et $\mathrm{al}^{14}$ estimate that averting $1 \mathrm{HIV}$ infection saves $\delta=23$ DALYs. To decide if the use of the gel is cost-effective, we compare the cost of preventing 1 person from being infected with HIV with the life-time cost of keeping 1 person on ART, and we also compare the cost of saving 1 DALY with the per capita gross national income per year.

\section{RESULTS}

The model provides a good fit to data from seroprevalence surveys in South Africa, and the predicted trends in HIV prevalence, incidence, and mortality are shown in Figure 1. We assume that the microbicide is rolled-out starting in 2012 and is available throughout South Africa by 2015 . HIV incidence falls rapidly as gel use increases (Fig. 1, centre), and the highest level of impact is seen by 2015. Prevalence falls less rapidly as transmission declines (Fig. 1, left), and mortality falls more slowly still (Fig. 1, right) because of the average life expectancy of 11 years for people who are infected with HIV and are not on ART; the full impact on mortality is not seen until 2030.

Between 2011 and 2020, low coverage (25\%) of the microbicide would avert 0.25 (0.02 to 0.38$)$ million incident cases and prevent 0.05 (0.01 to 0.07$)$ million deaths, whereas high coverage (90\%) would avert 1.10 (0.06 to 2.04$)$ million cases and prevent 0.20 ( 0.01 to 0.35$)$ million deaths (Table 1$)$. In the following 10 years (2021-2030), the impact on incidence would be only slightly greater, but the impact on mortality would be much greater. Low coverage of the microbicide would still avert 0.25 (0.02 to 0.39$)$ million incident cases but would prevent 0.24 (0.02 to 0.37$)$ million deaths, whereas high coverage would avert 1.23 (0.06 to 2.59) million cases and prevent 1.10 (0.06 to 2.07) million deaths.

In the CAPRISA 004 trial, $^{9}$ the annual incidence in the placebo arm was $I=9.1 \%(6.9 \%$ to $11.7 \%)$. The women in the trial had an average of 5 sexual encounters per month, with the number decreasing from 8.5 to 3.0 per month at the end of the trial, so that $\eta \approx 60 \pm 12$ per year. Fitting our model to the epidemic in South Africa, the reduction in incidence under the high coverage scenario is $65 \%$ of the reduction in transmission, whereas at low coverage, it is $53 \%$ of the reduction in transmission so that $\phi=0.65$ and 0.53 in the 2 scenarios. Substituting these values in equation 2 , the cost per infection averted at 90\% coverage is US \$1701 ( \pm US \$1281), whereas at $25 \%$ coverage, it is US \$2392 ( \pm US\$1830), and with equation 3, the cost per DALY averted at 90\% coverage is US \$74 ( \pm US \$56) and at 25\% coverage, is US \$104 ( \pm US \$77) (Table 2).

\section{DISCUSSION}

Widespread use of the vaginal microbicide, tenofovir gel, as part of prevention programs, could lead to significant reductions in the rate of new HIV infections and AIDS-related mortality. In South Africa, high coverage (tenofovir gel used in 
FIGURE 1. Trends in prevalence, incidence, and mortality resulting from the rollout of a vaginal microbicide program between 2012 and 2015. The model is fitted to the trend in HIV prevalence among adults of age 15 years or more. Black line (immediately above the shaded areas): no intervention. Blue lines: microbicide is used in $90 \%$ (high), $50 \%$ (medium), and 25\% (low) of all sex acts. Shaded areas give the $95 \%$ confidence limits.
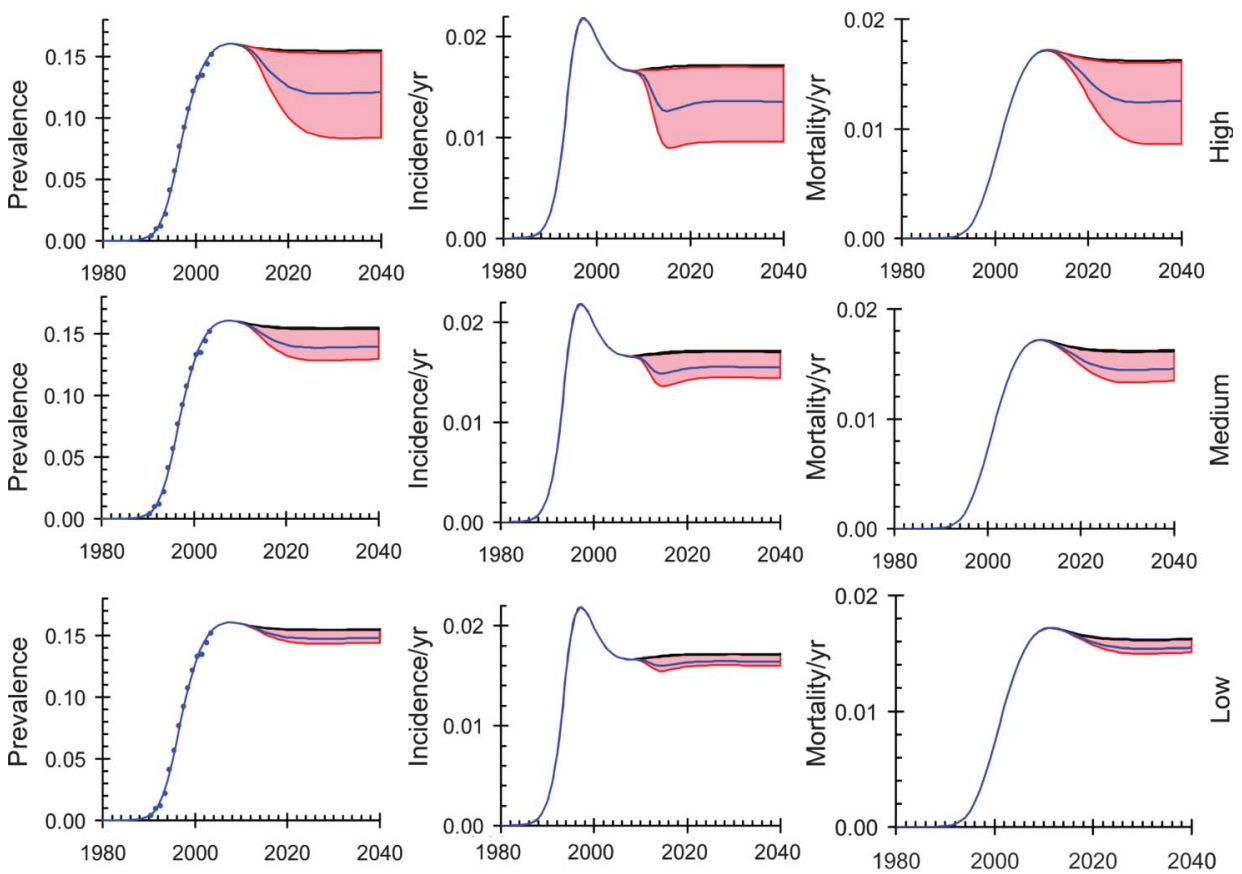

$90 \%$ of sex encounters) could avert 2.33 ( 0.12 to 4.63 ) million new infections and save 1.30 (0.07 to 2.42) million lives over the next 20 years in South Africa. In a low-coverage scenario (tenofovir gel used in $25 \%$ of sex encounters), it could avert 0.50 (0.04 to 0.77$)$ million new infections and save $0.29(0.02$ to 0.44 ) million deaths over 20 years in South Africa.

At low coverage, the cost of preventing 1 person from being infected with HIV is $28 \%$ of the life-time cost of providing ART to 1 person which Verguet et al estimate to be US $\$ 8396,{ }^{14}$ whereas the cost of saving 1 DALY is only $2 \%$ of the per capita gross national income per year, ${ }^{15}$ which in South Africa is $\$ 5770 .{ }^{16}$ Condom social marketing, the use of sterile needles, female condom use by sex workers, ART for the prevention of mother-to-child transmission, blood safety, sexually transmitted infection treatment, peer education, and male circumcision were investigated by Verguet et $\mathrm{al}^{14}$ and their cost-effectiveness lay between US \$5/DALY for condom social marketing in Chad and US \$194/DALY for the use of sterile needles in South Africa. ${ }^{17}$ The cost-effectiveness of using tenofovir gel microbicides at about US \$100 per DALY saved (Table 2), compares favourably with these other interventions. In this analysis, the cost of the microbicide is US $\$ 0.60$ per sexual encounter, but it is likely that this could be significantly reduced especially if the cost of the applicator and the wrapping can be reduced. Providing women with access to this microbicide should be cost-saving and cost-effective. None of the estimates allow for the savings in treatment and hospitalization costs, which will be incurred by HIV-positive people before they die or start ART and which will further improve the cost-benefit ratio.

In countries where the incidence of HIV infection is lower than it is in southern Africa, the cost-effectiveness will be less favourable because the cost per infection averted rises as the incidence falls (equation 1). In countries such as Senegal, where the prevalence and the incidence are about one-tenth of that in South Africa, tenofovir gel would be less cost-effective if used in the general population. However, if gel use can be targeted at women at highest risk, such as commercial

TABLE 1. The Reduction in Prevalence (at the end of each decade) and in the Cumulative Incidence and Deaths (over each decade)

\begin{tabular}{|c|c|c|c|c|}
\hline \multirow[b]{2}{*}{ Period } & \multirow[b]{2}{*}{ Measure } & \multicolumn{3}{|c|}{ Coverage } \\
\hline & & $90 \%$ & $50 \%$ & $25 \%$ \\
\hline \multirow[t]{3}{*}{2011 to 2020} & Prevalence & $0.93(0.05$ to 1.72$)$ & 0.44 (0.03 to 0.72$)$ & 0.21 (0.01 to 0.32$)$ \\
\hline & Incidence & $1.10(0.06$ to 2.04$)$ & $0.53(0.03$ to 0.85$)$ & $0.25(0.02$ to 0.38$)$ \\
\hline & Deaths & $0.20(0.01$ to 0.35$)$ & $0.10(0.01$ to 0.16$)$ & $0.05(0.01$ to 0.07$)$ \\
\hline \multirow[t]{2}{*}{2021 to 2030} & Prevalence & 1.19 (0.06 to 2.42$)$ & $0.54(0.03$ to 0.90$)$ & $0.25(0.02$ to 0.38$)$ \\
\hline & Incidence & $1.23(0.06$ to 2.59$)$ & $0.56(0.03$ to 0.93$)$ & $0.25(0.02$ to 0.39$)$ \\
\hline \multirow{2}{*}{2011 to 2030} & Incidence & $2.33(0.12$ to 4.63$)$ & $1.09(0.06$ to 1.78$)$ & $0.50(0.04$ to 0.77$)$ \\
\hline & Deaths & $1.30(0.07$ to 2.42$)$ & $0.62(0.04$ to 1.01$)$ & $0.29(0.02$ to 0.44$)$ \\
\hline
\end{tabular}

Data are given in columns assuming that the microbicide is used in $90 \%, 50 \%$, and $25 \%$ of sex acts. Numbers are in millions and numbers in brackets give $95 \%$ confidence limits. 
TABLE 2. The Cost Effectiveness of the Use of a Vaginal Microbicide at Low and High Coverage

\begin{tabular}{lcccc}
\hline Coverage & $\begin{array}{c}\text { US \$/Infection } \\
\text { Averted }\end{array}$ & $\begin{array}{c}\text { US \$/Infection } \\
\text { Not Averted }\end{array}$ & $\begin{array}{c}\text { US \$/DALY } \\
\text { Saved }\end{array}$ & $\begin{array}{c}\text { US \$ Gross } \\
\text { National Income }\end{array}$ \\
\hline $25 \%$ & $2392(1830)$ & 8396 & $104(77)$ & 5770 \\
$90 \%$ & $1701(1281)$ & & $74(56)$ & \\
\hline
\end{tabular}

Numbers in brackets are $95 \%$ confidence limits. The cost per infection not averted is the estimated cost of keeping a person on ART for 23 years. ${ }^{14}$

sex workers, the impact of the gel on HIV-incidence would be increased. Now that the effectiveness of tenofovir gel to prevent HIV infection has been demonstrated, we hope that further research into new drugs and new delivery methods will lead to lower costs with even greater efficacy.

The CAPRISA 004 trial included information, counselling, condom promotion, and treatment of sexually transmitted infections for all study participants. Hence, the trial's effectiveness level estimates, which we have used in this analysis, provide an incremental measure of adding tenofovir gel to an existing prevention program. This highlights the need for tenofovir gel to be implemented as part of a combination prevention approach.

The model we used is not stratified on age and sex but captures the most important features of the impact of vaginal microbicides on the transmission of HIV. A model structured on age and sex would provide further insights into the way in which microbicide use can best be targeted at those women at highest risk. For example, the incidence of HIV in women in South Africa peaks at the age of about 25 years, and close to $60 \%$ of all new infections in women are among those aged between 20 and 30 years $^{18}$ so that targeting women in this age group, at least initially, could yield substantial benefits.

The model did not include tenofovir resistance because the CAPRISA 004 trial showed no evidence of tenofovir resistance in women who became infected with HIV in the CAPRISA 004 trial. Resistance may, however, become an important consideration over time as tenofovir use for AIDS treatment becomes widespread. Further, the model did not include any impact of tenofovir gel on female-to-male transmission, as there is currently no evidence for this.

Although the factor $\phi$, which relates the reduction in population level incidence to the reduction in the individual risk of infection, depends on the details of the epidemic and the structure of the model, it is unlikely to differ greatly for countries in East and southern Africa where the epidemic pattern is similar to that in South Africa. If epidemics can be brought close to elimination, formally with $R_{0}$ close to 1 , a small reduction in individual risk can lead to a much greater proportional reduction in population level incidence so that the cost-effectiveness would be correspondingly greater.

The concept of combination prevention draws upon the idea that multiple prevention interventions can achieve synergy and thereby reduce HIV incidence to a greater extent than possible with each singly. The synergy goal of combination prevention is critical for the eventual reversal in the current upward trend in the HIV epidemic. Because young women have the highest HIV incidence rates in the southern African epidemic, high coverage of tenofovir gel in this group in combination with high coverage of circumcision in men plus education, HIV testing, and condom promotion could achieve the synergy necessary to turn the tide of the epidemic in this region.

The most important limitation of this analysis lies in the uncertainty in the estimates of the extent to which gel use reduces transmission and further studies to confirm the results of the CAPRISA 004 trial, which should also provide better estimates of the impact, are urgently needed.

\section{REFERENCES}

1. Gouws E, for The International Group on Analysis of Trends in HIV Prevalence and Behaviours in Young People in Countries most Affected by HIV. Trends in HIV prevalence and sexual behaviour among young people aged 15-24 years in countries most affected by HIV. Sex Transm Infect. 2010;86(suppl 2):ii72-ii83.

2. UNAIDS. Report on the Global AIDS Epidemic. Geneva, Switzerland: Joint United Nations Programme on HIV/AIDS; 2008.

3. White RG, Orroth KK, Glynn JR, et al. Treating curable sexually transmitted infections to prevent HIV in Africa: still an effective control strategy? J Acquir Immune Defic Syndr. 2008;47:346-353.

4. Ross DA, Changalucha J, Obasi AI, et al. Biological and behavioural impact of an adolescent sexual health intervention in Tanzania: a community-randomized trial. AIDS. 2007;21:1943-1955.

5. Lissouba P, Taljaard D, Rech D, et al. A model for the roll-out of comprehensive adult male circumcision services in African low-income settings of high HIV incidence: the ANRS 12126 Bophelo Pele Project. PLoS Med. 2010; 7:e1000309.

6. Hallett TB, Alsallaq RA, Baeten JM, et al. Will circumcision provide even more protection from HIV to women and men? New estimates of the population impact of circumcision interventions. Sex Transm Infect. 2011; 87:88-93

7. Foss AM, Watts CH, Vickerman P, et al. Condoms and prevention of HIV. Br Med J. 2004;329:185-186.

8. Egger M. Outcomes of ART in resource-limited and industrialized countries. Paper presented at: 14th Conference on Retriviruses and Opportunistic Infections; Februray 25-28, 2007; San Francisco, CA.

9. Abdool Karim Q, Abdool Karim SS, Frohlich JA, et al. Effectiveness and safety of tenofovir gel, an antiretroviral microbicide, for the prevention of HIV infection in women. Science. 2011;329:1168-1174.

10. Abdool Karim SS, Churchyard GJ, Abdool Karim Q, et al. HIV infection and tuberculosis in South Africa: an urgent need to escalate the public health response. Lancet. 2009;374:921-933.

11. Gouws E, Stanecki KA, Lyerla R, et al. The epidemiology of HIV infection among young people aged 15-24 years in southern Africa. AIDS. 2008;22(suppl 4):S5-S16.

12. Williams BG, Lloyd-Smith JO, Gouws E, et al. The potential impact of male circumcision on HIV in sub-Saharan Africa. PLoS Med. 2006;3(7).

13. Anderson RM, May RM. Infectious Diseases of Humans: Dynamics and Control. Oxford, United Kingdom: Oxford University Press; 1991.

14. Verguet S, Walsh JA. Vaginal microbicides save money: a model of costeffectiveness in South Africa and the USA. Sex Transm Infect. 2010;86: 212-216.

15. Sachs J. Report of the Commission on Macroeconomics and Health. Geneva, Switzerland: World Health Organization; December 2001.

16. Farivari R, Chandrasekaran R, Chi Y, et al. World Development Indicators Database, Revised 9 July 2010. Washington, DC World Bank; 2010. Available at: http://siteresources.worldbank.org/DATASTATISTICS/ Resources/GNIPC.pdf. Accessed September 13, 2010.

17. Bertozzi S, Padian NS, Wegbreit J, et al. HIV/AIDS prevention and treatment. In: Jamison DT, ed. Disease Control Priorities in Developing Countries. Washington, DC; Oxford University Press and the World Bank; 2006.

18. Gouws E, Williams BG, Sheppard HW, et al. High incidence of HIV-1 in South Africa using a standardized algorithm for recent HIV seroconversion. J Acquir Immune Defic Syndr. 2002;29:531-535. 